\title{
Clinical outcomes of lithium disilicate glass-ceramic crowns fabricated with CAD/CAM technology: A systematic review
}

\section{Ocena kliniczna koron szklano-ceramicznych z dwukrzemianu litu wytwarzanych w technologii CAD/CAM - systematyczny przegląd piśmiennictwa}

\author{
Ahmed Aziz ${ }^{1, A-E}$, Omar El-Mowafy ${ }^{2, A-C, E, F}$, Saira Paredes ${ }^{3, A-C, E, F}$ \\ 1 Department of Preventive and Restorative Dentistry, College of Dental Medicine, University of Sharjah, UAE \\ ${ }^{2}$ Department of Restorative Dentistry, Faculty of Dentistry, University of Toronto, Canada \\ ${ }^{3}$ Private practice, Toronto, Canada \\ A - research concept and design; $B$ - collection and/or assembly of data; $C$ - data analysis and interpretation; \\ $D$ - writing the article; $E$ - critical revision of the article; $F$ - final approval of the article
}

Address for correspondence

Ahmed Aziz

Email: a.aziz@sharjah.ac.ae

Funding sources

None declared

Conflict of interest

None declared

Received on 0ctober 29, 2019

Reviewed on November 26, 2019

Accepted on December 19, 2019

Published online on June 30, 2020

Cite as

Aziz A, El-Mowafy 0, Paredes S. Clinical outcomes of lithium disilicate glass-ceramic crowns fabricated with CAD/CAM technology: A systematic review. Dent Med Probl. 2020;57(2):197-206. doi:10.17219/dmp/115522

D0I

$10.17219 / \mathrm{dmp} / 115522$

Copyright

(c) 2020 by Wroclaw Medical University

This is an article distributed under the terms of the

Creative Commons Attribution 3.0 Unported License (CC BY 3.0)

(https://creativecommons.org/licenses/by/3.0/).

\section{Abstract}

The use of ceramic materials and the computer-aided design/computer-aided manufacturing (CAD/CAM) technology for the fabrication of complete-coverage restorations has significantly increased in the last decade. The aim of this study was to evaluate the survival rate of anterior and posterior monolithic and bilayered lithium disilicate glass-ceramic (LDGC) CAD/CAM crowns, and to identify the types of complications associated with the main clinical outcomes reported in clinical trials. MEDLINE/PubMed, Embase, Scopus, Web of Science, Cochrane Library, and ClinicalTrials.gov were searched by 2 independent reviewers for clinical studies published between 2006 and 2019, following the Preferred Reporting Items for Systematic Reviews and Meta-Analyses (PRISMA) statement. The electronic search was supplemented by a hand search. Quality assessment for the included studies was performed. Qualitative and quantitative data was extracted from each study. Out of 219 studies, 6 studies that evaluated LDGC CAD/CAM crowns were identified and used for data extraction. The included studies had 154 participants, who received 204 crowns.

The short- to medium-term survival and success rates were high. Biological complications occurred more frequently than technical complications. No esthetic complications were reported. This review indicated that the medium-term survival rate of LDGC CAD/CAM crowns was high. Further multicenter studies with longer follow-ups and larger sample sizes are needed in order to augment the data already in existence.

Key words: survival, glass-ceramic, prosthodontics, dental porcelain, computer-aided design/computeraided manufacturing

Słowa kluczowe: przetrwanie, szklano-ceramiczne, protetyka stomatologiczna, ceramika stomatologiczna, komputerowo wspomagane projektowanie i produkcja 


\section{Introduction}

The advance of digital dentistry and a high demand for metal-free restorations have led to rapid evolution in processing technologies as well as to the development of newer restorative materials. Several clinical studies have demonstrated that all-ceramic crowns, particularly lithium disilicate glass-ceramic (LDGC), may be a potentially promising alternative to metalbased crowns. ${ }^{1-9}$

Lithium disilicate glass-ceramic materials are available as pressable (IPS ${ }^{\circledR}$ e.max Press; Ivoclar Vivadent AG, Schaan, Liechtenstein) and machinable for the computer-aided design/computer-aided manufacturing (CAD/CAM) processing technology (IPS e.max CAD; Ivoclar Vivadent). Since it was introduced in 2006 , the latter has become widely used because of its excellent performance both in vitro and in vivo. ${ }^{10,11}$ Due to its improved translucency and good mechanical properties, IPS e.max CAD can be used as a core material with the layering technique or as a fully anatomical restoration. ${ }^{12,13}$ Lithium disilicate glass-ceramic monolithic blocks have become the material of choice for chairside systems due to their high strength and greater translucency as compared to other ceramic blocks. ${ }^{14}$ However, the use of LDGC crows in the bilayered arrangement has not gained popularity due to a weak link between the core and the veneering porcelain, which caused pre-mature failure. IPS e.max CAD demonstrates excellent biomechanical characteristics, as it exhibits a flexural strength of $360 \mathrm{MPa}$ after complete crystallization. ${ }^{15}$ A significant increase in the strength of the restoration takes place when an adhesive cement is used rather than a conventional one, as concluded in an in-vitro study done in $2006 .^{16}$

The blocks are manufactured by the continuous pressure casting of transparent glass ingots to obtain a defect-free block without pores or pigments. The blocks are made available for milling in the CAD/CAM machine in a partially crystallized, 'soft' state to minimize the damage to the material caused by the wear of the milling diamond burs. ${ }^{17}$ IPS e.max CAD blocks are composed of 0.2-1.0-micrometer lithium metasilicate crystals $\left(\mathrm{Li}_{2} \mathrm{SiO}_{3}\right)(40.0 \%$ by volume); blocks in this state appear blue-violet. These crystals give the material favorable processing properties, a moderately high strength and a high edge stability. After milling, the restoration undergoes a 2 -stage firing process for $<20 \mathrm{~min}$ in order to complete the crystallization process, during which lithium disilicate crystals $\left(\mathrm{Li}_{2} \mathrm{Si}_{2} \mathrm{O}_{5}\right)$ are formed, giving the restoration its final shade and the desired high strength. The resulting ceramic is composed of fine grains of size $1.5 \mu \mathrm{m}$ and $70.0 \%$ of crystals by volume.

Due to its reliable performance, the LDGC material is indicated for several types of dental restorations, such as inlays, onlays, veneers, crowns, and implantsupported crowns. ${ }^{11}$ Several clinical studies have reported the clinical performance and survival rate of LDGC CAD/CAM crowns. ${ }^{1-9}$ A systematic review needs to be conducted in order to combine the findings from these studies and to establish overall conclusions about their clinical performance. Therefore, the aim of this systematic review was to evaluate the survival rate of anterior and posterior monolithic and bilayered LDGC crowns manufactured with CAD/CAM systems as well as to identify the types of clinical complications reported in clinical trials.

\section{Material and methods}

This review was conducted in accordance with the Preferred Reporting Items for Systematic Reviews and MetaAnalyses (PRISMA) statement. ${ }^{18}$

\section{Eligibility criteria}

A PICOS question was used to identify studies in this review: P (population) - patients who received ceramic crowns; I (intervention) - crowns made of glass ceramic; $\mathrm{C}$ (comparison) - not applicable in this study; O (outcomes) - survival rate and type of complication; S (study type) - randomized controlled trials (RCTs) and clinical follow-up studies. The inclusion and exclusion criteria are listed in Table 1.

Table 1. Inclusion and exclusion criteria

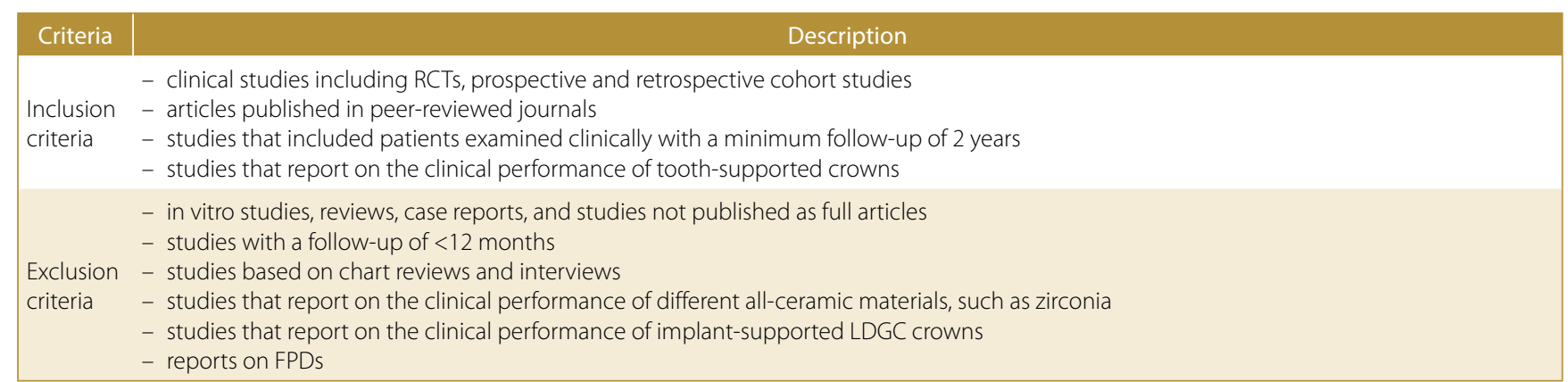

RCT - randomized clinical trial; LDGC - lithium disilicate glass-ceramic; FPD - fixed partial denture. 


\section{Information sources and search strategy}

Several electronic databases, including MEDLINE/ PubMed, Embase, Scopus, Web of Science, and Cochrane Library were searched to identify clinical trials performed to evaluate the clinical performance of LGDC crowns fabricated with CAD/CAM systems. The search was carried out from January 2006 (the year in which IPS e.max CAD was introduced) until June 2019, and it was limited to human studies and the English language only. The search was extended to find unpublished and ongoing clinical trials using ClinicalTrials.gov (www.clinicaltrials. gov). The search strategies defined for the databases described above are listed in Appendix 1. The appropriate search terms for the electronic search were key words, Medical Subject Headings (MeSH) and subject headings. The electronic search was further supplemented by the manual search of 4 different journals: The International Journal of Prosthodontics, Journal of Prosthodontics, Journal of Prosthetic Dentistry, and Journal of Esthetic and Restorative Dentistry.

Appendix 1. Search strategy

\section{PubMed}

1 lithium disilicate.mp. (578)

2 Crowns/ (15475)

3 Dental Restoration Failure/ (7474)

4 dental restoration, permanent/ or inlays/ (22529)

5 "glass ceramic*.mp. [mp=title, abstract, original title, name of substance word, subject heading word, floating sub-heading word, keyword heading word, protocol supplementary concept word, rare disease supplementary concept word, unique identifier, synonyms] (1811)

6 "crown*..mp. [mp=title, abstract, original title, name of substance word, subject heading word, floating sub-heading word, keyword heading word, protocol supplementary concept word, rare disease supplementary concept word, unique identifier, synonyms] (36366)

7 (dental adj2 restor*).mp. [mp=title, abstract, original title, name of substance word, subject heading word, floating sub-heading word, keyword heading word, protocol supplementary concept word, rare disease supplementary concept word, unique identifier, synonyms] (30249)

8 "clinical performance".mp. [mp=title, abstract, original title, name of substance word, subject heading word, floating sub-heading word, keyword heading word, protocol supplementary concept word, rare disease supplementary concept word, unique identifier, synonyms] (6738)

9 failure.mp. [mp=title, abstract, original title, name of substance word, subject heading word, floating sub-heading word, keyword heading word, protocol supplementary concept word, rare disease supplementary concept word, unique identifier, synonyms] (740680)

10 success.mp. [mp=title, abstract, original title, name of substance word, subject heading word, floating sub-heading word, keyword heading word, protocol supplementary concept word, rare disease supplementary concept word, unique identifier, synonyms] (212925)

11 survival.mp. [mp=title, abstract, original title, name of substance word, subject heading word, floating sub-heading word, keyword heading word, protocol supplementary concept word, rare disease supplementary concept word, unique identifier, synonyms] (1003959)

12 treatment outcome.mp. or exp Treatment Outcome/ (909585)

13 exp Dental Porcelain/ (10050)

14 Lithium Compounds/ (2759)

151 or 5 or 13 or $14(13806)$

163 or 8 or 9 or 10 or 11 or 12 (2470226)

17 longevity.mp. (35015)

18 "all ceramic".mp. (1712)

1916 or $17(2495410)$

2015 or 18 (14260)

21 "CAD CAM".mp. (2268)

22 exp Computer-Aided Design/ (16406)

2321 or $22(16808)$

242 or 4 or 6 or 7 (63551)

2519 and 20 and 24 (1829)

2623 and 25 (401)

27 limit 26 to (clinical study or clinical trial, all or clinical trial, phase i or clinical trial, phase ii or clinical trial, phase iii or clinical trial, phase iv or clinical trial or controlled clinical trial or multicenter study or pragmatic clinical trial or randomized controlled trial) (56)

\section{Embase}

1 lithium disilicate.mp. (667)

2 "glass ceramic*".mp. [mp=title, abstract, heading word, drug trade name, original title, device manufacturer, drug manufacturer, device trade name, keyword, floating subheading word, candidate term word] (2087)

3 "crown*".mp. [mp=title, abstract, heading word, drug trade name, original title, device manufacturer, drug manufacturer, device trade name, keyword, floating subheading word, candidate term word] (55906)

4 (dental adj2 restor*).mp. [mp=title, abstract, heading word, drug trade name, original title, device manufacturer, drug manufacturer, device trade name, keyword, floating subheading word, candidate term word] (6613)

5 "clinical performance".mp. [mp=title, abstract, heading word, drug trade name, original title, device manufacturer, drug manufacturer, device trade name, keyword, floating subheading word, candidate term word] (9743)

6 failure.mp. [mp=title, abstract, heading word, drug trade name, original title, device manufacturer, drug manufacturer, device trade name, keyword, floating subheading word, candidate term word] (1388858)

7 success.mp. [mp=title, abstract, heading word, drug trade name, original title, device manufacturer, drug manufacturer, device trade name, keyword, floating subheading word, candidate term word] (354138)

8 survival.mp. [mp=title, abstract, heading word, drug trade name, original title, device manufacturer, drug manufacturer, device trade name, keyword, floating subheading word, candidate term word] (1535084)

9 treatment outcome.mp. or exp Treatment Outcome/ (1472690)

10 CAD CAM.mp. or exp Computer-Aided Design/ (24107)

11 exp Dental Porcelain/ (1768)

12 longevity.mp. (43666)

13 "all ceramic."mp. (1874)

14 exp tooth crown/ (20916)

15 exp dental restoration/ (59432)

16 exp lithium derivative/ (4817)

171 or 2 or 11 or 13 (5184)

185 or 6 or 7 or 8 or 9 or 12 (4066948)

19 ("emax" or"e.max").mp. [mp=title, abstract, heading word, drug trade name, original title, device manufacturer, drug manufacturer, device trade name, keyword, floating subheading word, candidate term word] (4898)

2017 or $19(9613)$

2116 or $20(14287)$

223 or 4 or 14 or $15(110597)$

2310 and 18 and 20 and 22 (266)

24 limit 23 to (clinical trial or randomized controlled trial or controlled clinical trial or multicenter study or phase 1 clinical trial or phase 2 clinical trial or phase 3 clinical trial or phase 4 clinical trial) (36)

Scopus

( ( TITLE-ABS-KEY ("lithium disilicate") OR TITLE-ABS-KEY ("glass cerami**") OR TITLE-ABS-KEY ("dental ceramic*" $))$ ) AND ((TITLE-ABS-KEY ("crown*"))) AND ( (TITLE-ABS-KEY ("clinical performance") OR TITLE-ABS-KEY (failure) OR TITLE-ABS-KEY ( success) OR TITLE-ABS-KEY (longevity) OR TITLE-ABSKEY ( survival )) ) AND ( TITLE-ABS-KEY ( "CAD CAM")) AND ( LIMIT-TO ( DOCTYPE, "ar") OR LIMIT-TO (DOCTYPE, "re")) 


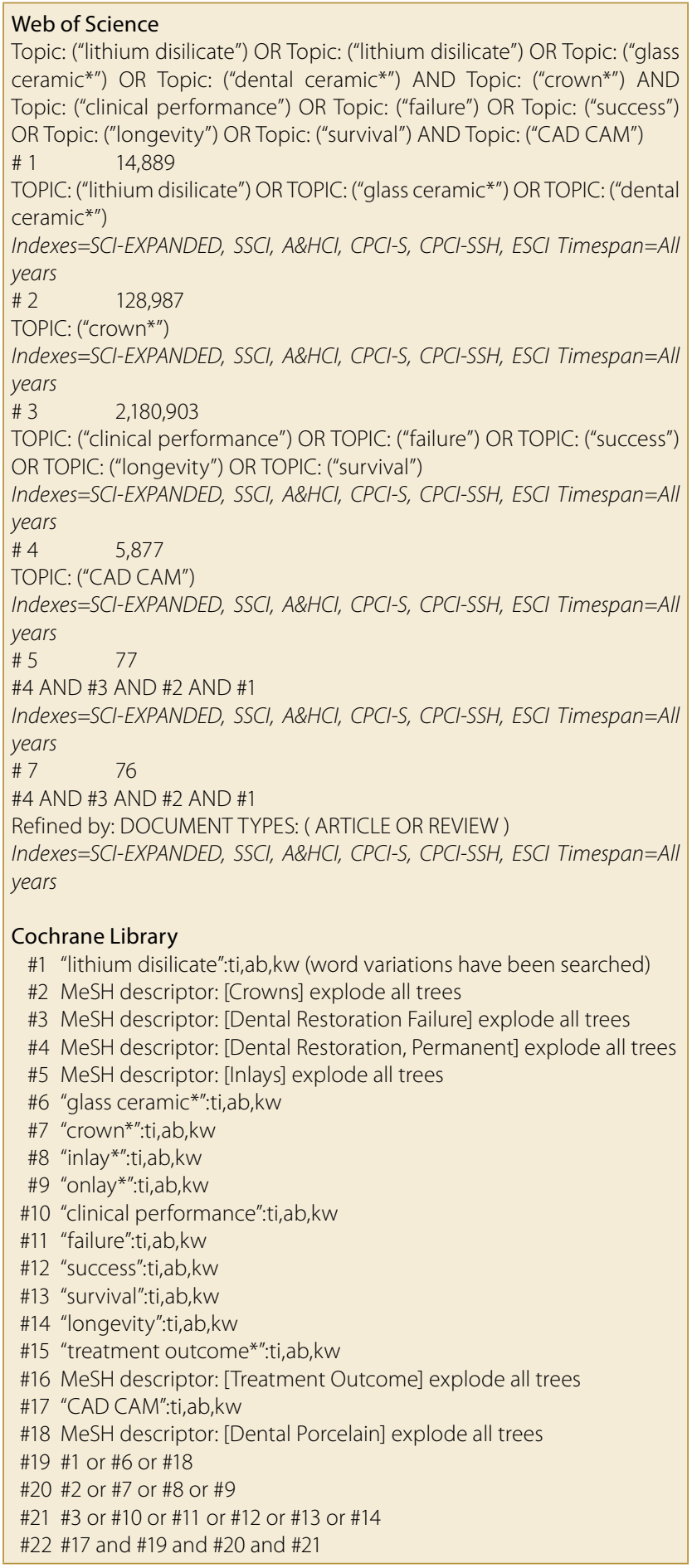

\section{Study selection}

The electronic search was conducted systematically by 2 independent investigators at multiple stages. The $1^{\text {st }}$ stage involved screening the relevant titles after removing duplicated studies; any disagreements were resolved through a discussion. At the $2^{\text {nd }}$ stage, the abstracts of the selected titles were independently analyzed and any disagreements were resolved through a discussion. At the final stage, the full texts of the studies of possible relevance were obtained. The manual search of the references of the selected articles was performed to supplement the electronic search. The inter-reviewer agreement of 0.86 was calculated using Cohen's kappa coefficient at the title and abstract stages.

\section{Quality assessment in individual studies}

The quality assessment of the selected studies was carried out according to the methods described by Hayashi et al. ${ }^{19}$ Each study was evaluated following 26 criteria and a percentage value was calculated, representing the quality of the study (Appendix 2).

Appendix 2. Quality assessment criteria according to the methods described by Hayashi et al. ${ }^{19}$

\begin{tabular}{|c|c|}
\hline Item & Question \\
\hline 1 & Is the hypothesis / aim / objective of the study clearly described? \\
\hline 2 & Is the setting of the study or the source of the subjects described? \\
\hline 3 & Is the distribution of the study population by age or gender described? \\
\hline 4 & Are the inclusion criteria stated? \\
\hline 5 & Are the exclusion criteria stated? \\
\hline 6 & Are the kinds of treatment well described? \\
\hline 7 & $\begin{array}{l}\text { Are the main outcomes to be measured clearly described } \\
\text { in the Introduction or Methods section? }\end{array}$ \\
\hline 8 & Is the sample size stated? \\
\hline 9 & Was the sample size justified? \\
\hline 10 & Was the concurrent control group used? \\
\hline 11 & Was random allocation to treatment used? \\
\hline 12 & Was the method of random allocation given? \\
\hline 13 & Was the blind assessment of the outcome carried out? \\
\hline 14 & Was there more than 1 examiner for outcome assessment? \\
\hline 15 & Was the examiner calibration carried out? \\
\hline 16 & Are the statistical methods described? \\
\hline 17 & Is the participation/follow-up rate stated? \\
\hline 18 & Was the participation/follow-up rate greater than $80 \% ?$ \\
\hline 19 & Are the non-participants/subjects lost to follow-up described? \\
\hline 20 & Are the main findings of the study clearly described? \\
\hline 21 & $\begin{array}{l}\text { Are the results stated in absolute numbers when feasible } \\
\text { (e.g., 10/20, not 50\%)? }\end{array}$ \\
\hline 22 & Are confidence intervals given? \\
\hline 23 & Are any important adverse events reported? \\
\hline 24 & Are any conclusions stated? \\
\hline 25 & Was this a prospective study? \\
\hline 26 & Was the ethical approval obtained? \\
\hline
\end{tabular}

\section{Data collection}

The following qualitative data was extracted from the definitive list: author(s), year of publication, type of study, study setting, number of operators providing the crowns, number of evaluators and their independency, reported follow-up period, assessment criteria used for each study, tooth type (anterior or posterior), type of crown 
(monolithic vs layered), and method of fabrication (chairside vs laboratory). The following quantitative data was extracted: number of patients, drop-out rate, participants' age range, number of crowns, number of crowns made in the university setting vs private practice or both, condition of the restored teeth (vital vs non-vital), location of the crowns (anterior, premolar or molar), type of cement, number of complications, type of complications - biological (caries, pulpal involvement, tooth fracture), technical (framework fracture, chipping, debonding) or esthetic (color mismatch, marginal discoloration) - and success and survival rates.

In this review, success was defined as crowns that were present without core fracture, porcelain fracture, caries, periodontal inflammation, or endodontic signs and symptoms. ${ }^{20}$ Survival was defined as crowns having remained in situ, with or without modification during the entire period of observation; these were referred to as "survived crowns". 21 After the data had been collected, no metaanalysis was performed due to qualitative differences across the studies and insufficient data.

\section{Results}

\section{Study selection}

The primary electronic search resulted in a total number of 219 studies. After eliminating duplicates, and screening the studies at the title and abstract levels, 12 studies were carried forward for full-text assessment from the electronic search and 1 article from hand searching. Out of these 13 studies, 7 studies were excluded, and the remaining 6 studies were included and used for data extraction. The process of identifying eligible articles is described in Fig. 1.
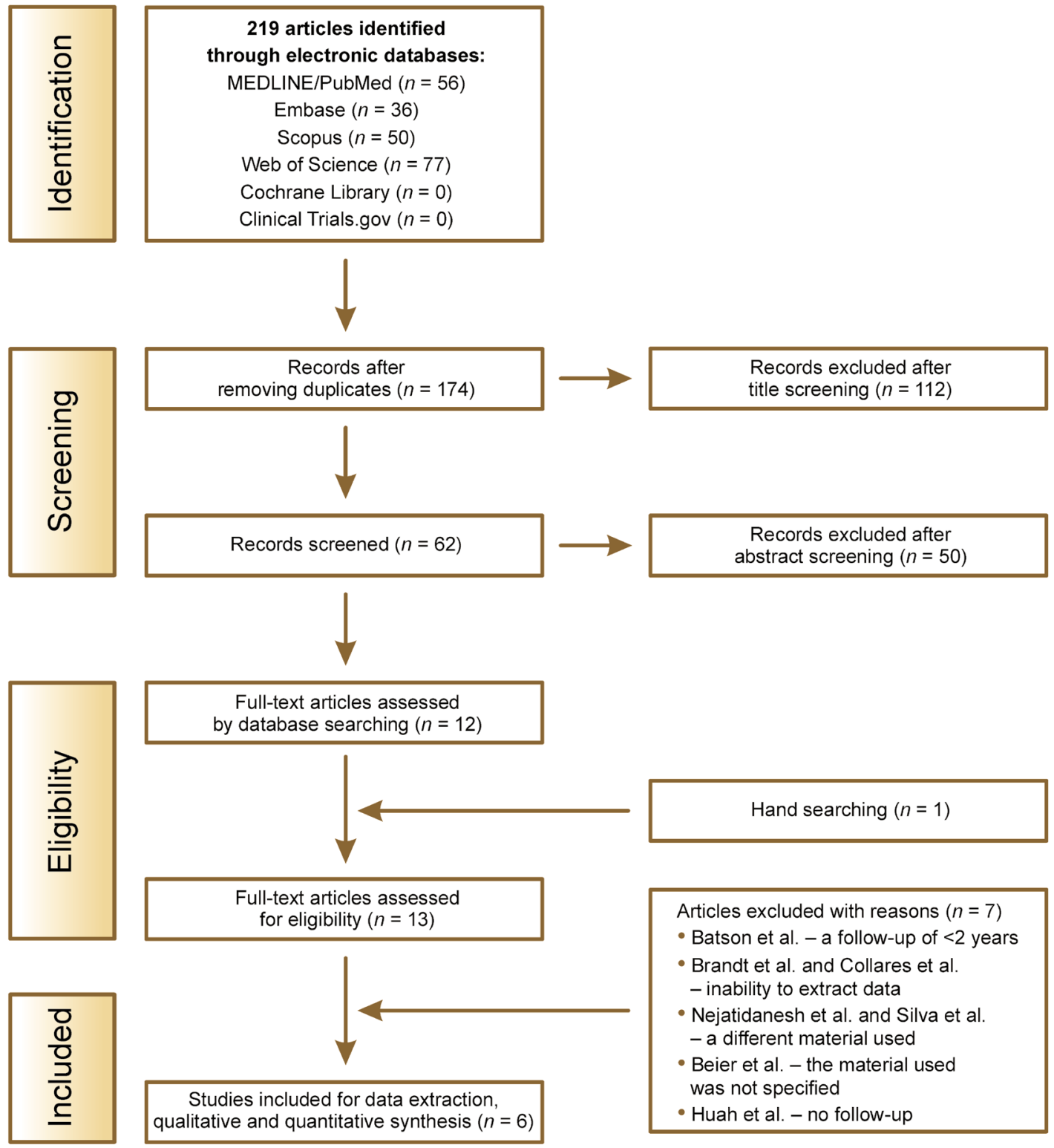

Full-text articles assessed by database searching $(n=12)$

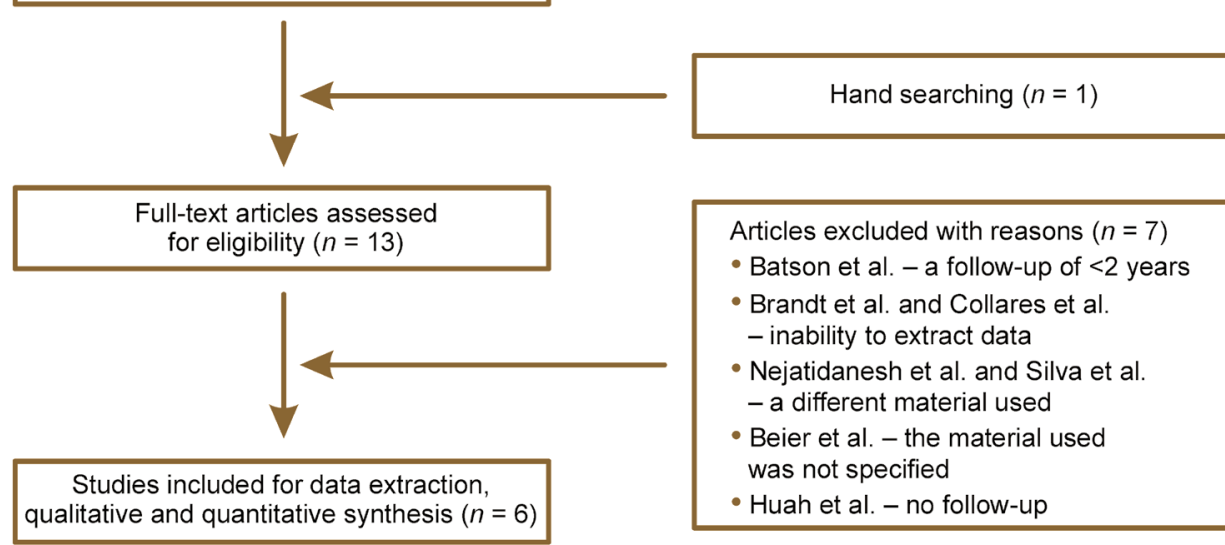

Fig. 1. Flowchart of the study selection process 
Appendix 3. Results of the quality assessment of the included studies

\begin{tabular}{|c|c|c|c|c|c|c|c|c|c|c|c|c|c|c|c|c|c|c|c|c|c|c|c|c|c|c|c|}
\hline \multirow{2}{*}{ Study } & \multicolumn{26}{|c|}{ Items assessed according to Appendix 2} & \multirow{2}{*}{$\begin{array}{c}\text { Percentage } \\
\text { of'yes' } \\
\text { answers } \\
{[\%]}\end{array}$} \\
\hline & 1 & 2 & 3 & 4 & 5 & 6 & 7 & 8 & 9 & 10 & 11 & 12 & 13 & 14 & 15 & 16 & 17 & 18 & 19 & 20 & 21 & 22 & 23 & 24 & 25 & 26 & \\
\hline $\begin{array}{l}\text { Fasbinder et al. }{ }^{1} \\
2010\end{array}$ & $x$ & $x$ & - & $x$ & - & $x$ & $x$ & $x$ & - & - & - & - & $x$ & X & - & - & $x$ & $x$ & $x$ & $x$ & $x$ & - & $x$ & $x$ & $x$ & $x$ & 65.4 \\
\hline $\begin{array}{l}\text { Cortellini and Canale } \\
2012\end{array}$ & $x$ & $x$ & $x$ & $x$ & $x$ & $x$ & $x$ & $x$ & - & $x$ & $x$ & $x$ & - & - & - & $x$ & - & - & - & $x$ & $x$ & - & $x$ & $x$ & $x$ & - & 65.4 \\
\hline $\begin{array}{l}\text { Seydler and Schmitter } \\
2015\end{array}$ & $x$ & $x$ & $x$ & $x$ & $x$ & $x$ & $x$ & $x$ & $x$ & $x$ & $x$ & $x$ & - & - & - & $x$ & - & - & - & $x$ & $x$ & - & $x$ & $x$ & $x$ & $x$ & 73.1 \\
\hline $\begin{array}{l}\text { Akın et al. }{ }^{8} \\
2015\end{array}$ & $x$ & $x$ & $x$ & $x$ & $x$ & $x$ & $x$ & $x$ & $x$ & $x$ & $x$ & - & - & $x$ & $x$ & $x$ & $x$ & $x$ & - & $x$ & $x$ & - & $x$ & $x$ & $x$ & $x$ & 84.6 \\
\hline $\begin{array}{l}\text { Rauch et al. }{ }^{5} \\
2018\end{array}$ & $x$ & $x$ & $x$ & $x$ & $x$ & $x$ & $x$ & $x$ & $x$ & $x$ & $x$ & $x$ & $x$ & $x$ & $x$ & $x$ & $x$ & - & $x$ & $x$ & $x$ & $x$ & $x$ & $x$ & $x$ & $x$ & 96.2 \\
\hline $\begin{array}{l}\text { Aziz et al. }{ }^{9} \\
2019\end{array}$ & $x$ & $x$ & $x$ & $x$ & $x$ & $x$ & $x$ & $x$ & $x$ & - & - & - & $x$ & $x$ & $x$ & $x$ & $x$ & - & $x$ & $x$ & $x$ & $x$ & $x$ & $x$ & - & $x$ & 80.8 \\
\hline
\end{tabular}

$X$ - answer'yes'.

Table 2. Characteristics of the included studies

\begin{tabular}{|c|c|c|c|c|c|c|c|c|c|}
\hline Study & Study design & Setting & $\begin{array}{c}\text { Method } \\
\text { of } \\
\text { fabrication }\end{array}$ & $\begin{array}{l}\text { Type } \\
\text { of crown }\end{array}$ & $\begin{array}{l}\text { Tooth } \\
\text { type }\end{array}$ & $\begin{array}{l}\text { No. of } \\
\text { operators }\end{array}$ & $\begin{array}{c}\text { No. of } \\
\text { evaluators }\end{array}$ & $\begin{array}{c}\text { Observation } \\
\text { period } \\
\text { [months] }\end{array}$ & $\begin{array}{c}\text { Evaluation } \\
\text { criteria }\end{array}$ \\
\hline $\begin{array}{l}\text { Fasbinder et al. }{ }^{1} \\
2010\end{array}$ & prospective & university & chairside & monolithic & posterior & 1 & $\begin{array}{c}2 \\
\text { independent }\end{array}$ & 24 & $\begin{array}{l}\text { modified } \\
\text { USPHS }\end{array}$ \\
\hline $\begin{array}{l}\text { Cortellini and Canale } \\
2012\end{array}$ & prospective & private practice & laboratory & monolithic & posterior & 1 & $\begin{array}{l}\text { the same } \\
\text { operator }\end{array}$ & 42 & NR \\
\hline $\begin{array}{l}\text { Akın et al. }{ }^{8} \\
2015\end{array}$ & $\mathrm{RCT}$ & university & laboratory & layered & anterior & 1 & $\begin{array}{c}1 \\
\text { independent }\end{array}$ & 24 & $\begin{array}{c}\text { modified } \\
\text { USPHS }\end{array}$ \\
\hline $\begin{array}{l}\text { Rauch et al. }{ }^{5} \\
2018\end{array}$ & prospective & $\begin{array}{c}\text { university/ } \\
\text { private practice }\end{array}$ & chairside & monolithic & posterior & 4 & $\begin{array}{c}2 \\
\text { independent }\end{array}$ & 120 & $\begin{array}{l}\text { modified } \\
\text { USPHS }\end{array}$ \\
\hline $\begin{array}{l}\text { Aziz et al. }{ }^{9} \\
2019\end{array}$ & retrospective & university & chairside & monolithic & posterior & $\begin{array}{l}\text { dental } \\
\text { students }\end{array}$ & $\begin{array}{c}2 \\
\text { independent }\end{array}$ & 48 & $\begin{array}{l}\text { modified } \\
\text { CDA }\end{array}$ \\
\hline
\end{tabular}

USPHS - United States Public Health Service; CDA - California Dental Association; NR - not reported.

Table 3. Quantitative data extracted from the included studies

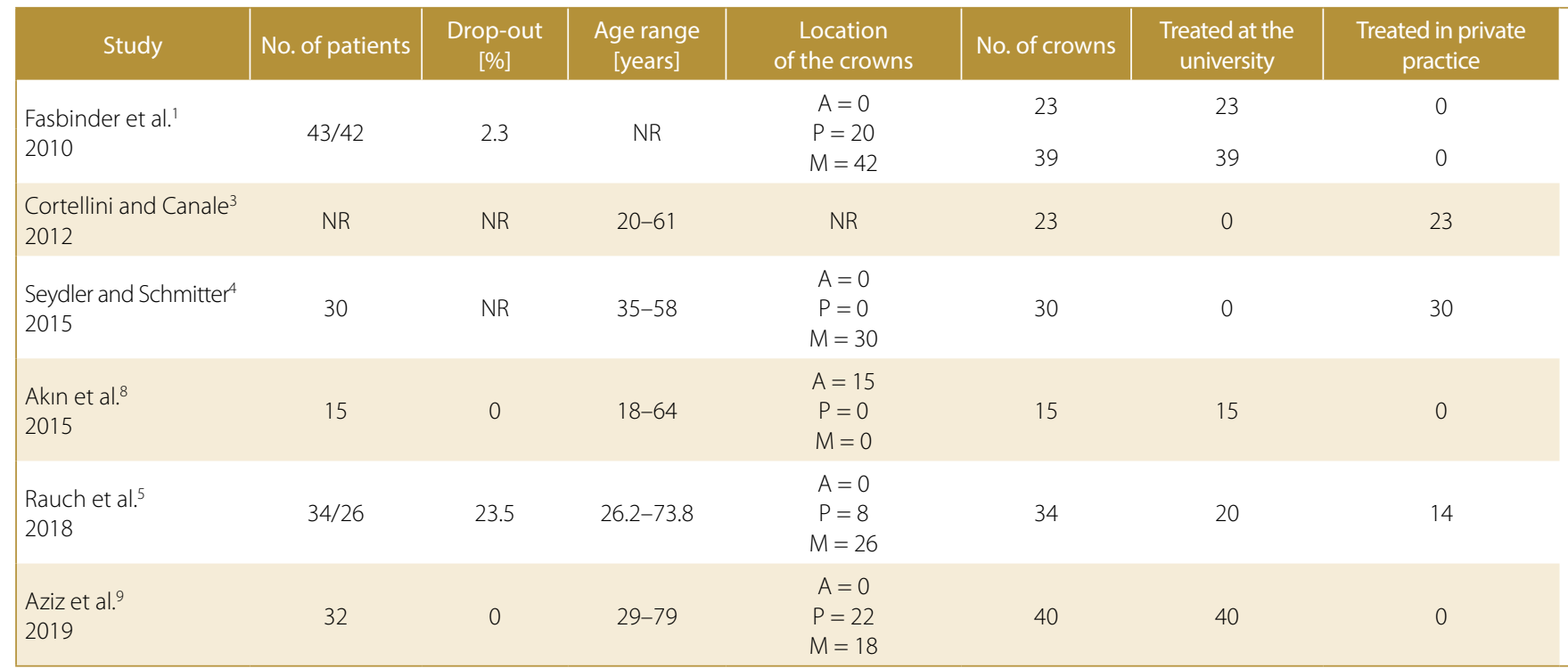

A - anterior teeth; P - premolar teeth; M - molar teeth; NR - not reported. 


\section{Results of quality assessment}

According to the criteria of quality assessment, the studies in this review involved results ranging from $65.4 \%$ to $96.2 \%$ (Appendix 3).

\section{Data collection and the characteristics of the studies}

The included studies were published between 2010 and 2019. Five studies evaluated LDGC CAD/CAM crowns prospectively, ${ }^{1,3-5,8} 1$ study was an $\mathrm{RCT}^{8}$ and 1 - a retrospective study. ${ }^{9}$ Of the studies presenting results derived from the same sample, only the most recent study was included. ${ }^{2,5-7}$ Five studies reported on the clinical performance of posterior monolithic LDGC CAD/CAM crowns ${ }^{1,3-5,9}$ and 1 study, by Akın et al., reported on anterior layered crowns. ${ }^{8}$ Four studies used the chairside technique ${ }^{1,4,5,9}$ and 2 used the laboratory method of fabrication. ${ }^{3,8}$ The characteristics of the studies are presented in Table 2. The quantitative data related to the clinical performance of LDGC CAD/CAM (IPS e.max CAD) crowns are presented in Table 3.

In the included studies, a total of 204 crowns were inserted in 154 patients and evaluated for a period ranging from 2 to 10 years. A total of 9 participants were lost to follow-up from all the studies combined. All the studies reported the number of participants who received LDGC CAD/CAM crowns and the location of the crowns (anterior, premolar or molar) except the study by Cortellini and Canale. ${ }^{3}$ Two studies reported the distribution of the crowns in the maxilla or the mandible. ${ }^{4,9}$ Using the pooled data, it was found that there was a total of 14 complications in 204 crowns that had been placed, representing a complication rate of $6.9 \%$ (Table 4 ). Nine crowns were considered as failed and 5 were considered as survived. There were more biological $(n=10)$ than technical complications $(n=4)$. The most common biological failure reported was an endodontic complication $(n=5)$, followed by secondary caries $(n=4)$ and tooth fracture $(n=1)$. In Rauch et al.s study, the tooth extracted due to an apical infection was counted as an endodontic complication and the tooth fractured due to caries was counted as a recurrent caries complication. ${ }^{5}$ Four technical complications were reported - 1 debonding, 1 crown fracture and 2 open margins. The most frequently reported complications occurred in the molar teeth, but it was not possible to determine whether there was any particular predilection for failure in the maxilla vs the mandible, as there was not enough information available to make a definitive conclusion. No esthetic complications such as color mismatch or marginal discoloration were reported.

The survival rate reported by Fasbinder et al., ${ }^{1}$ Cortellini and Canale, ${ }^{3}$ and Akın et al. ${ }^{8}$ was $100.0 \%$, as no complications were reported over a follow-up period of 2-3.5 years. In Seydler and Schmitter's study, the survival rate was stipulated from the survival curve analysis (93.3\%), as they counted the 2 endodontic complications as failed rather than survived crowns after 2 years. ${ }^{4}$ Rauch et al. reported a survival rate of $85.3 \%$ after 10 years. ${ }^{5}$ Except for the studies performed by Rauch et al. ${ }^{5}$ and Aziz et al., ${ }^{9}$ none of the others actually reported the overall success rates. The succes rates presented in Table 3 were calculated as the percentage of crowns without complications.

\begin{tabular}{|c|c|c|c|c|c|}
\hline Vital & Non-vital & $\begin{array}{l}\text { Type of } \\
\text { cement }\end{array}$ & $\begin{array}{c}\text { No. } \\
\text { of complications }\end{array}$ & $\begin{array}{c}\text { Success rate } \\
{[\%]}\end{array}$ & $\begin{array}{c}\text { Survival rate } \\
{[\%]}\end{array}$ \\
\hline NR & NR & Multilink ${ }^{\circledR}$ Automix; Ivoclar Vivadent AG & 0 & 100.0 & 100.0 \\
\hline NR & NR & experimental cement; Ivoclar Vivadent AG & 2 & 94.9 & 100.0 \\
\hline NR & NR & Multilink Automix; Ivoclar Vivadent AG, Variolink ${ }^{\circledR}$ Veneer; Ivoclar Vivadent AG & 0 & 100.0 & 100.0 \\
\hline 14 & 16 & Multilink Automix; Ivoclar Vivadent AG & 2 & 93.3 & 93.3 \\
\hline NR & NR & Variolink II; Ivoclar Vivadent AG & 0 & 100.0 & 100.0 \\
\hline 17 & 17 & Multilink Sprint; Ivoclar Vivadent AG & 9 & 73.5 & 85.3 \\
\hline 7 & 33 & RelyX $X^{\circledR}$ Unicem; 3M ESPE, Calibra ${ }^{\circledR}$ Universal; Dentsply Sirona & 3 & 92.5 & 95.0 \\
\hline
\end{tabular}


Table 4. Summary of all complications reported for LDGC computer-aided design/computer-aided manufacturing (CAD/CAM) crowns

\begin{tabular}{|c|c|c|c|c|}
\hline Study & Complication & $\begin{array}{c}\text { Category } \\
\text { of complication }\end{array}$ & $\begin{array}{l}\text { Period } \\
\text { [months] }\end{array}$ & Outcome \\
\hline \multirow{2}{*}{$\begin{array}{l}\text { Seydler and Schmitter } \\
2015\end{array}$} & endodontic complication & biological & 12 & failed \\
\hline & endodontic complication & biological & 12 & failed \\
\hline \multirow{9}{*}{$\begin{array}{l}\text { Rauch et al. }{ }^{5} \\
2018\end{array}$} & endodontic complication & biological & at baseline & survived \\
\hline & endodontic complication & biological & 13.2 & survived \\
\hline & recurrent caries & biological & 24 & failed \\
\hline & recurrent caries & biological & 48 & survived \\
\hline & tooth extraction due to an apical infection & biological & 72 & failed \\
\hline & tooth fracture due to recurrent caries & biological & 72 & failed \\
\hline & tooth fracture & biological & 84 & failed \\
\hline & debonded crown & technical & 24 & survived \\
\hline & crown fracture & technical & 33.6 & failed \\
\hline \multirow{3}{*}{$\begin{array}{l}\text { Aziz et al. }{ }^{9} \\
2019\end{array}$} & recurrent caries & biological & 24 & survived \\
\hline & open margin & technical & 30 & failed \\
\hline & open margin & technical & 37 & failed \\
\hline Total & 14 & $\begin{array}{l}10 \text { biological } \\
4 \text { technical }\end{array}$ & - & $\begin{array}{l}5 \text { survived } \\
9 \text { failed }\end{array}$ \\
\hline
\end{tabular}

\section{Discussion}

The present study aimed at establishing scientific evidence regarding the efficacy of LDGC CAD/CAM crowns. Only published clinical trials were included. Conference abstracts and studies published by the manufacturer were excluded, because it was not possible to extract the required data from them. The sample size was relatively small for all the included studies. However, since they were mostly prospective clinical trials, the known difficulty of recruiting more patients and maintaining a low drop-out rate are always a limitation inherent to this type of study.

There was no significant association between the incidences of failure and the type of cement. This could be explained by a reliable bond that could be achieved with resin cements. The group of crowns cemented with an experimental cement in Fasbinder et al's study was excluded from the analysis, since the cement was withdrawn from the market, and therefore, findings with this particular cement would be irrelevant to the main goals of this investigation. ${ }^{1}$

None of the included studies reported any association between the incidence of complications and the tooth type (premolar vs molar). In fact, the location of the crowns (premolars vs molars) was not correlated with failure or success in most investigations with the exception of 1 study, where it was stated that most complications occurred in the molar teeth. ${ }^{5}$ Unfortunately, this report did not provide enough data for our investigation to make meaningful conclusions regarding the impact of this factor. ${ }^{5}$ Moreover, none of the studies reported whether there were any differences in the occurrence of complications when comparing the restorations placed in the maxilla vs the mandible. Similarly, there was no evident statement whether the setting in which the crowns were placed (university vs private practice) had an impact on survival. There were no evident differences that could be found regarding the survival of the crowns placed on vital or non-vital teeth. This particular issue was not even reported in the studies included in our review.

None of the included studies compared the clinical outcome of LDGC crowns to the "gold standard", that being metal-ceramic crowns. In the study by Cortellini and Canale, LDGC CAD/CAM crowns $(n=23)$ were compared to 212 pressed crowns for a period of 42 months. ${ }^{3}$ No complication was reported for the CAD/CAM group and only 1 crown fractured in the pressed group after 3 years. ${ }^{3}$ In the study conducted by Seydler and Schmitter, the performance of 30 monolithic LDGC CAD/CAM crowns was compared to 30 veneered zirconia crowns. ${ }^{4}$ The number of biological complications was similar for both groups; 2 endodontic complications occurred in each. No technical complications such as fracture, chipping or cracks were noted for either group after 2 years of follow-up. ${ }^{4}$

This systematic review demonstrated that biological complications occurred more often than technical complications. The need for endodontic treatment after the cementation of crowns can be attributed to the overreduction of the natural tooth structure, especially in young patients, in addition to continuous pulpal trauma. The incidence of recurrent secondary caries could not be linked to the status of oral hygiene due to insufficient information, but could be related to an increased marginal gap 
in all-ceramic crowns. Akın et al. compared the marginal and internal adaptation of $15 \mathrm{LDGCCAD/CAM}$ crowns to 15 heat-pressed LDGC crowns (IPS. e.max Press, Ivoclar Vivadent AG); there was no statistically significant difference between the 2 fabrication techniques. ${ }^{8}$ The median marginal gap for the CAD/CAM group was within the clinically acceptable range $(132.2 \mu \mathrm{m})$, but it was reported as low as $71.0 \mu \mathrm{m}$. Also, no recurrent caries was detected in their study due to a small sample size and a short observation time of 2 years, which was not sufficient for carious lesions to develop. ${ }^{8}$ Caries at the crown margin was reported in 1 study, and occurred in 2 teeth. ${ }^{5}$ The mean marginal gap measured in that study was $81.0 \mu \mathrm{m}$, but the actual marginal gap in the crowns affected by recurrent caries was not disclosed, and therefore, it was not possible to make any solid conclusions regarding the contribution of the marginal gap to the occurrence of recurrent caries. ${ }^{5}$ Although such a marginal gap is considered to be within the clinically acceptable range, it might be concluded that even within this range, secondary caries could be detected if a good oral hygiene level was not maintained. ${ }^{22}$ However, LDGC crowns demonstrated similar recurrent caries rate and fewer endodontic problems as compared to metal-ceramic crowns after 5 years, and it could be assumed that the level of oral hygiene was most likely the same in both study populations. ${ }^{23}$

With regard to technical complications, 1 fracture and 1 case of debonding occurred in the same study. ${ }^{5}$ The authors reported that the crown fractured due to the insufficient occlusal reduction of the lower second molar, whereas the other crown debonded due to inadequate retention, as the tooth height was $<3.0 \mathrm{~mm} .{ }^{5}$ These technical complications could likely have been avoided had the manufacturer's recommendations been followed carefully. No crown chipping was reported due to the absence of veneering porcelain in monolithic crowns, which is usually associated with this type of complication. Even in the case of the layered crowns evaluated in 1 study, there was no chipping or fracture after 2 years. ${ }^{8}$ However, this could be attributed to a short-term follow-up and to the location of the crowns in the anterior region, where the occlusal forces are minimal. ${ }^{8}$ Two crowns provided by dental students presented with open margins. ${ }^{9}$ No esthetic complications were reported. The color match of LDGC CAD/CAM crowns was rated as being good to excellent in all studies, and the patients were very satisfied with the esthetic appearance of the crowns.

Apart from the clinical findings reported here, this review also showed that there was a limited number of studies with relatively small sample sizes that focused on the clinical performance of LDGC CAD/CAM crowns. There is a need for clinical trials evaluating both fabrication techniques (chairside and laboratory) with larger sample sizes, longer follow-up times and blinded evaluators. This is highly important, as it will considerably enrich the currently available data and will help to identify with more confidence the clinical performance of LDGC crowns fabricated using both methods of manufacturing. It is also recommended that, in order to provide more useful data, studies should be designed to include the distribution of complications with regard to the tooth type, tooth location, tooth condition (vital or non-vital), study setting (university and/or private practice), and the experience level of the operator(s).

\section{Conclusions}

Based on the findings and within the limitations of this systematic review, it can be concluded that the short- to medium-term survival rate of LDGC CAD/CAM crowns was high (from $93.3 \%$ to $100 \%$ ). Biological complications were the most frequently reported sequelae and mostly occurred in the first 2 years after the cementation of the crowns, regardless of the type of cement used.

\section{ORCID iDs}

Ahmed Aziz (1) https://orcid.org/0000-0001-9724-8376

Omar El-Mowafy (1) https://orcid.org/0000-0002-3301-3911

Saira Paredes (i) https://orcid.org/0000-0003-2165-9560

\section{References}

1. Fasbinder DJ, Dennison JB, Heys D, Neiva G. A clinical evaluation of chairside lithium disilicate CAD/CAM crowns: A two-year report. J Am Dent Assoc. 2010;141(Suppl 2):S10-S14.

2. Reich S, Fischer S, Sobotta B, Klapper HU, Gozdowski S. A preliminary study on the short-term efficacy of chairside computer-aided design/computer-assisted manufacturing-generated posterior lithium disilicate crowns. Int J Prosthodont. 2010;23(3):214-216.

3. Cortellini D, Canale A. Bonding lithium disilicate ceramic to featheredge tooth preparations: A minimally invasive treatment concept. $J$ Adhes Dent. 2012;14(1):7-10.

4. Seydler B, Schmitter M. Clinical performance of two different CAD/ CAM-fabricated ceramic crowns: 2-year results. J Prosthet Dent. 2015;114(2):212-216.

5. Rauch A, Reich S, Dalchau L, Schierz O. Clinical survival of chair-side generated monolithic lithium disilicate crowns: 10-year results. Clin Oral Investig. 2018;22(4):1763-1769.

6. Reich S, Schierz O. Chair-side generated posterior lithium disilicate crowns after 4 years. Clin Oral Investig. 2013;17(7):1765-1772.

7. Rauch A, Reich S, Schierz O. Chair-side generated posterior monolithic lithium disilicate crowns: Clinical survival after 6 years. Clin Oral Investig. 2017;21(6):2083-2089.

8. Akın A, Toksavul S, Toman M. Clinical marginal and internal adaptation of maxillary anterior single all-ceramic crowns and 2-year randomized controlled clinical trial. J Prosthodont. 2015;24(5):345-350.

9. Aziz A, El-Mowafy O, Tenenbaum HC, Lawrence HP, Shokati B. Clinical performance of chairside lithium disilicate glass-ceramic CADCAM crowns. J Esthet Restor Dent. 2019;31(6):613-619.

10. Giordano R. Materials for chairside CAD/CAM-produced restorations. J Am Dent Assoc. 2006;137(Suppl):14S-21S.

11. Poticny DJ, Klim J. CAD/CAM in-office technology: Innovations after 25 years for predictable, esthetic outcomes. J Am Dent Assoc. 2010;141(Suppl 2):5S-9S.

12. Wolfart S, Eschbach S, Scherrer S, Kern M. Clinical outcome of threeunit lithium-disilicate glass-ceramic fixed dental prostheses: Up to 8 years results. Dent Mater. 2009;25(9):e63-e71.

13. Etman MK, Woolford MJ. Three-year clinical evaluation of two ceramic crown systems: A preliminary study. J Prosthet Dent. 2010;103(2):80-90. 
14. Duret F, Preston JD. CAD/CAM imaging in dentistry. Curr Opin Dent. 1991;1(2):150-154.

15. Guess PC, Schultheis S, Bonfante EA, Coelho PG, Ferencz JL, Silva NRFA. All-ceramic systems: Laboratory and clinical performance. Dent Clin North Am. 2011;55(2):333-352.

16. Bindl A, Lüthy $H$, Mörmann $W H$. Thin-wall ceramic CAD/CAM crown copings: Strength and fracture pattern. J Oral Rehabil. 2006;33(7):520-528.

17. Coelho Santos G Jr, Moraes Coelho Santos MJ Jr, Rizkalla AS, Madani DA, El-Mowafy O. Overview of CEREC CAD/CAM chairside system. Gen Dent. 2013;61(1):36-40;quiz 41

18. Moher D, Liberati A, Tetzlaff J, Altman DG; PRISMA Group. Preferred reporting items for systematic reviews and meta-analyses: The PRISMA statement. PLoS Med. 2009;6(7):e1000097.

19. Hayashi M, Wilson NHF, Yeung CA, Worthington HV. Systematic review of ceramic inlays. Clin Oral Investig. 2003;7(1):8-19.

20. Patel DR, O'Brien T, Petrie A, Petridis H. A systematic review of outcome measurements and quality of studies evaluating fixed toothsupported restorations. J Prosthodont. 2014;23(6):421-433.

21. Pjetursson BE, Sailer I, Zwahlen $M$, Hämmerle $\mathrm{CH}$. A systematic review of the survival and complication rates of all-ceramic and metal-ceramic reconstructions after an observation period of at least 3 years. Part I: Single crowns. Clin Oral Implants Res. 2007;18(Suppl 3):73-85.

22. Matta RE, Schmitt J, Wichmann M, Holst S. Circumferential fit assessment of CAD/CAM single crowns - a pilot investigation on a new virtual analytical protocol. Quintessence Int. 2012;43(9):801-809.

23. Sailer I, Makarov NA, Thoma DS, Zwahlen M, Pjetursson BE. Allceramic or metal-ceramic tooth-supported fixed dental prostheses (FDPs)? A systematic review of the survival and complication rates. Part I: Single crowns (SCs). Dent Mater. 2015;31(6):603-623. 